Home Search Collections Journals About Contact us My IOPscience

Dense ionic fluids confined in planar capacitors: in- and out-of-plane structure from classical density functional theory

This content has been downloaded from IOPscience. Please scroll down to see the full text.

2016 J. Phys.: Condens. Matter 28244007

(http://iopscience.iop.org/0953-8984/28/24/244007)

View the table of contents for this issue, or go to the journal homepage for more

Download details:

IP Address: 131.211.104.35

This content was downloaded on 22/02/2017 at 10:17

Please note that terms and conditions apply.

You may also be interested in:

Fundamental measure theory for the electric double layer: implications for blue-energy harvesting and water desalination

Andreas Härtel, Mathijs Janssen, Sela Samin et al.

Time-dependent density functional theory for ion diffusion in electrochemical systems

Jian Jiang, Dapeng Cao, De-en Jiang et al.

Anisotropy and memory during cage breaking events close to a wall

Matthias Kohl, Andreas Härtel and Michael Schmiedeberg

Coarse-grained simulations of an ionic liquid-based capacitor: I. Density, ion size, and valency

effects

Konrad Breitsprecher, Peter Košovan and Christian Holm

Non-monotonic effect of confinement on the glass transition

Fathollah Varnik and Thomas Franosch

Can ionophobic nanopores enhance the energy storage capacity of electric-double-layer capacitors containing nonaqueous electrolytes?

Cheng Lian, Honglai Liu, Douglas Henderson et al. 


\title{
Dense ionic fluids confined in planar capacitors: in- and out-of-plane structure from classical density functional theory
}

\author{
Andreas Härtel ${ }^{1,2}$, Sela Samin ${ }^{2}$ and René van Roij ${ }^{2}$ \\ 1 Institute of Physics, Johannes Gutenberg-University Mainz, Staudinger Weg 9, 55128 Mainz, Germany \\ 2 Institute for Theoretical Physics, Center for Extreme Matter and Emergent Phenomena, \\ Utrecht University, Leuvenlaan 4, 3584 CE Utrecht, The Netherlands \\ E-mail: AnHaerte@uni-mainz.de
}

Received 5 December 2015, revised 11 February 2016

Accepted for publication 22 February 2016

Published 26 April 2016

\begin{abstract}
The ongoing scientific interest in the properties and structure of electric double layers (EDLs) stems from their pivotal role in (super)capacitive energy storage, energy harvesting, and water treatment technologies. Classical density functional theory (DFT) is a promising framework for the study of the in- and out-of-plane structural properties of double layers. Supported by molecular dynamics simulations, we demonstrate the adequate performance of DFT for analyzing charge layering in the EDL perpendicular to the electrodes. We discuss charge storage and capacitance of the EDL and the impact of screening due to dielectric solvents. We further calculate, for the first time, the in-plane structure of the EDL within the framework of DFT. While our out-of-plane results already hint at structural in-plane transitions inside the EDL, which have been observed recently in simulations and experiments, our DFT approach performs poorly in predicting in-plane structure in comparison to simulations. However, our findings isolate fundamental issues in the theoretical description of the EDL within the primitive model and point towards limitations in the performance of DFT in describing the out-of-plane structure of the EDL at high concentrations and potentials.
\end{abstract}

Keywords: density functional theory, electric double layer, in-plane structure, ionic liquid, differential capacitance, capacitive deionization, supercapacitor

(Some figures may appear in colour only in the online journal)

\section{Introduction}

Nowadays, the efficient storage of electric charges is more important than ever due to our continuously increasing need for electric energy. For this reason, (super)capacitors have attracted large interest in recent years [1,2]. Their electrodes $[1,3]$, can be utilized for the construction of sustainable energy-conversion [4-8] and capacitive deionization $[9,10]$ technology. All these devices exploit the capacitive properties of the electric double layers (EDLs), which are established in the vicinity of electrode surfaces by ionic charges, drawn from the ionic electrolytes the electrodes are immersed in [11]. Accordingly, many studies have focused on the detailed description of the EDL and its properties during the last years [12-21].
In a recent study, Merlet and co-workers proposed an explanation for some non-linear response in the differential capacitance of a parallel plate capacitor by analyzing the inplane structure of the EDL by means of molecular dynamics (MD) simulations [22, 23]. They found a voltage-dependent structural transition in the first ionic layer of a common ionic liquid $\left(\mathrm{BMI}-\mathrm{PF}_{6}\right)$ in contact with the electrodes, which they constructed from carbon particles. Further simulation studies have verified hints for this voltage-dependent structural transition [19, 24, 25] and, recently, also experimental studies on the structure of EDLs have been performed [26, 27]. However, a detailed theoretical description beyond these and older work on interfaces [28] is still missing.

A promising microscopic theory, based on fundamental statistical physics, is classical density functional theory (DFT). 
Originally introduced for electronic systems in 1964 [29, 30], the framework has also been adopted and applied to classical systems [31-33], especially in the field of soft condensed matter [34-36], but also in (commercial) tools for gas sorption data analysis of porous materials [37-39]. The DFT framework of fundamental measure theory (FMT) [40-42] has been shown to provide a quantitative benchmark for the important model system of hard spheres [43], where it resolved the long-standing question on the interfacial free energy in the crystal-fluid interface [44]. Even more, FMT accurately predicts pair correlations in confined, dense, and asymmetric, mixtures of hard spheres [45].

In contrast, a comparably successful functional for the primitive model of charged (asymmetric) hard spheres is still missing. While charges have been incorporated into DFT in several forms [17, 36, 46-52], their description typically lacks the correct treatment of the interplay between Coulombic and steric contributions, as known from the mean spherical approximation in bulk [53-57]. Thus, the incorporation of charges into DFT is of ongoing scientific interest and involves fundamental issues such as testing the contact value theorem $[58,59]$ and analyzing the decay of correlations [60, 61], but is also of relevance for devices with charged interfaces such as supercapacitors.

In this work, we apply DFT in order to investigate the fundamental properties of the in- and out-of-plane structure of the EDL. To ensure a theoretical description of particle ordering in our primitive model, we apply the White Bear mark II fundamental measure functional [62] for the hard-sphere contributions, which has been shown to provide excellent pair-correlation functions in uncharged systems [45]. Further, we solely add a mean-field Coulombic contribution, as described in previous work [63]. Since we extract pair correlations from DFT for the primitive model for the first time, we neglect the additional (approximate) correction discussed in previous work [63] in order to add only one new contribution to the well performing hard sphere functional. This procedure seems to be adequate for monovalent ions at room temperature [12], which we use in our primitive model of binary charged hard spheres, but now also at lower dielectric constants, i.e. at stronger Coulomb coupling.

In the next section, we explain in detail our theoretical framework of DFT and the extraction of correlation functions in the primitive model. Then, we discuss our choice of model and parameters, as well as the differences between DFT and simulations, in section 3. Turning to our results, we first apply our theory in section 4 to the out-of-plane order and test it against MD computer simulations. We further discuss the layering and adsorption of charges in the EDL, as well as its capacitance. Second, we apply our theory in section 5 for the first time to the in-plane order of the EDL in the inhomogeneous primitive model. We discuss our results in comparison with the simulations and conclude in section 6 .

\section{Structure in the primitive model}

\subsection{The primitive model}

We use the primitive model to describe an ionic liquid. In this model, sketched in figure 1, the ions of species $\nu$ are described

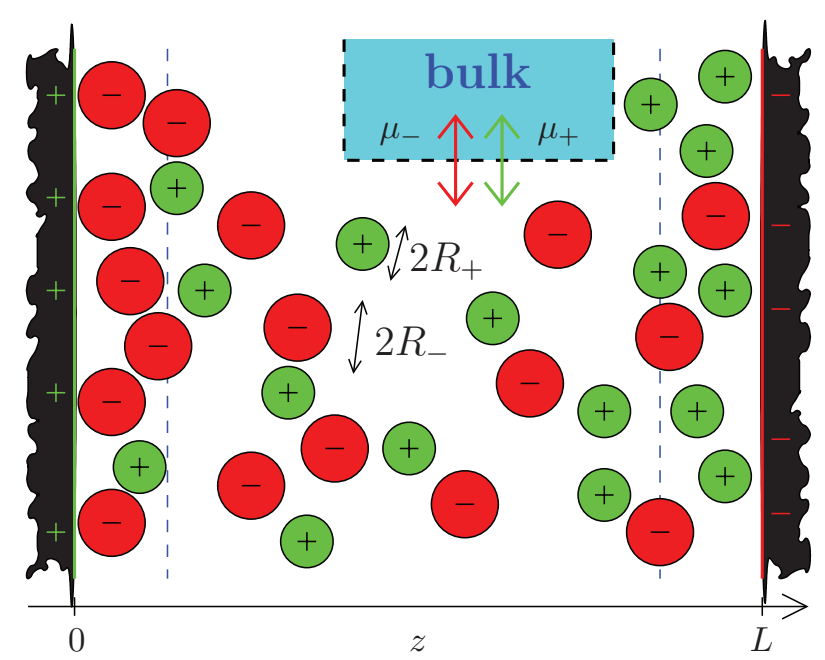

Figure 1. Sketch of the binary ionic liquid, confined in a parallel plate capacitor of plate separation $L$. The stroked lines illustrate the slab of the first layers of ions adjacent to the walls, defined by the first minima in the total concentration profiles $\rho(z)$. The grand canonical nature of the system is explained by the schematic connection to a bulk reservoir at fixed chemical potentials, which can exchange ions with the system.

as charged hard spheres with hard-sphere radius $R_{\nu}$ and charge valency $e Z_{\nu}$, where $e$ denotes the unit charge. Within the primitive model the solvent only enters as a dielectric background with relative permittivity $\varepsilon$ and temperature $T$. The interaction potential $\Phi_{\nu \nu^{\prime}}$ between two particles of species $\nu$ and $\nu^{\prime}$ with a core separation $r$ reads

$$
\Phi_{\nu \nu^{\prime}}(r)= \begin{cases}\infty & r<R_{\nu}+R_{\nu^{\prime}} ; \\ k_{\mathrm{B}} T \lambda_{\mathrm{B}} \frac{Z_{\nu} Z_{\nu^{\prime}}}{r} & r \geqslant R_{\nu}+R_{\nu^{\prime}},\end{cases}
$$

where $\lambda_{\mathrm{B}}=e^{2} /\left(4 \pi \varepsilon_{0} \varepsilon k_{\mathrm{B}} T\right)$ is the Bjerrum length in terms of the vacuum permittivity $\varepsilon_{0}$ and Boltzmann's constant $k_{\mathrm{B}}$. In an electrically neutral bulk with mean particle concentrations $\bar{\rho}_{\nu}$ for each species, this potential is typically screened within a distance characterized by the Debye length $\kappa^{-1}$, defined via $\kappa^{2}=4 \pi \lambda_{\mathrm{B}} \sum_{\nu} Z_{\nu}^{2} \bar{\rho}_{\nu}$

In our grand canonical ensemble (see figure 1) the mean particle concentrations $\bar{\rho}_{\nu}$ are related to the mean particle numbers $N_{\nu}$ in the total system volume $V$ via a spatial integration of the ensemble-averaged one-particle concentration (or density) profiles $\rho_{\nu}(\vec{r})$ of the respective species $\nu$ at positions $\vec{r}$ [36]. Consequently, the number of unit charges of species $\nu$ in a partial volume $V^{\prime} \subset V$ is

$$
Q_{\nu}\left(V^{\prime}\right)=\int_{V^{\prime}} Z_{\nu} \rho_{\nu}(\vec{r}) \mathrm{d} \vec{r} .
$$

In addition, we define the total number of positive and negative unit charges in the total system volume $V$ by $Q_{+}$and $Q_{-}$.

In our confined setting of a parallel plate capacitor, symmetry reduces the spatial parameters to only the cartesian $z$ component perpendicular to the hard capacitor walls (electrodes). These walls are located at $z=0$ and $z=L$, such that the concentration profile $\rho_{\nu}(z)$ of a species $\nu$ vanishes outside the interval $\left[R_{\nu}, L-R_{\nu}\right]$. This leads to species-dependent Stern 
layers of thickness $R_{\nu}$ (see also figure 1). The wall charges $e Q_{\mathrm{p}}$ and $e Q_{\mathrm{n}}$ on the respective positive and negative electrodes are distributed homogeneously, resulting in wall unit charge densities $\sigma_{\mathrm{p}}$ and $\sigma_{\mathrm{n}}$. Of course, electroneutrality requires $Q_{\mathrm{p}}+Q_{\mathrm{n}}+\sum_{\nu} Q_{\nu}=0$.

All charges in the system contribute to a (dimensionless) electrostatic potential $\phi$, which, for the ionic charge density $q(z)=\sum_{\nu} Z_{\nu} \rho_{\nu}(z)$, can be defined via Poisson's equation $\partial_{z}^{2} \phi(z)=-4 \pi \lambda_{\mathrm{B}} q(z)$ on the open interval $(0, L)$. The limits of the Poisson equation,

$$
\begin{gathered}
\lim _{z \rightarrow 0^{+}} \phi^{\prime}(z)=-4 \pi \lambda_{\mathrm{B}} \sigma_{\mathrm{p}}, \\
\lim _{z \rightarrow L^{-}} \phi^{\prime}(z)=4 \pi \lambda_{\mathrm{B}} \sigma_{\mathrm{n}}
\end{gathered}
$$

respect the electrode charges, which are uniquely determined via the ionic charge density $q(z)$ and the boundary values $\phi(0)=\beta e \Psi_{\mathrm{p}}$ and $\phi(L)=\beta e \Psi_{\mathrm{n}}$ of the electrostatic potential at the capacitor walls, where $\beta=\left(k_{\mathrm{B}} T\right)^{-1}$ defines an inverse temperature. Note that these equipotential surfaces, which arise from the system symmetries, set well-defined boundaries with no call for additional image-charge methods.

\subsection{Structure and correlations}

In statistical physics the three-dimensional partial static structure factors $S_{\nu \nu^{\prime}}(\vec{k})$ can be defined for the total number of particles $N=\sum_{\nu} N_{\nu}$ by [36]

$S_{\nu \nu^{\prime}}(\vec{k})=\frac{N_{\nu}}{N} \delta_{\nu \nu^{\prime}}+\frac{1}{N} \int_{V} \int_{V} \mathrm{e}^{-l \vec{k} \cdot\left(\vec{r}-\vec{r}^{\prime}\right)} \rho_{\nu}(\vec{r}) \rho_{\nu^{\prime}}\left(\vec{r}^{\prime}\right) h_{\nu \nu^{\prime}}^{(2)}\left(\vec{r}, \vec{r}^{\prime}\right) \mathrm{d} \vec{r} \mathrm{~d} \vec{r}^{\prime}$.

The partial structure factors $S_{\nu \nu^{\prime}}$ can be combined linearly to meaningful structure factors like the particle-particle (NN) structure $S_{\mathrm{NN}}(\vec{k})=\sum_{\nu} \sum_{\nu^{\prime}} S_{\nu \nu^{\prime}}(\vec{k})$, the particle-charge (NZ) structure $S_{\mathrm{NZ}}(\vec{k})=\sum_{\nu} \sum_{\nu^{\prime}} Z_{\nu^{\prime}} S_{\nu \nu^{\prime}}(\vec{k})$, and the charge-charge (ZZ) structure $S_{\mathrm{ZZ}}(\vec{k})=\sum_{\nu} \sum_{\nu^{\prime}} Z_{\nu} Z_{\nu^{\prime}} S_{\nu \nu^{\prime}}(\vec{k})[36,60]$. The last term of equation (5) involves the total correlation functions $h_{\nu \nu^{\prime}}^{(2)}$, which are related to the direct correlation functions via the Ornstein-Zernike relation [36]

$h_{\nu \nu^{\prime}}^{(2)}\left(\vec{r}, \vec{r}^{\prime}\right)=c_{\nu \nu^{\prime}}^{(2)}\left(\vec{r}, \vec{r}^{\prime}\right)+\sum_{\nu^{\prime \prime}=1}^{n} \int_{V} h_{\nu \nu^{\prime \prime}}^{(2)}\left(\vec{r}, \vec{r}^{\prime \prime}\right) \rho_{\nu^{\prime \prime}}\left(\vec{r}^{\prime \prime}\right) c_{\nu^{\prime \prime} \nu^{\prime}}^{(2)}\left(\vec{r}^{\prime \prime}, \vec{r}^{\prime}\right) \mathrm{d} \vec{r}^{\prime \prime}$

The static structure factor, as introduced in equation (5), is related to the total volume $V$, it is determined on. If we are interested in the structure of only the $N^{\text {slab }}=\sum_{\nu} N_{\nu}^{\text {slab }}$ ions which are located in the slab of length $L^{\text {slab }}$ next to the wall, we have to restrict our calculations to the corresponding set $\Gamma_{\nu}^{\text {slab }}$ of particles in the slab. Accordingly, we define the ensemble averaged partial in-plane structure factors in this slab by

$S_{\nu \nu^{\prime}}(\vec{q})=\left\langle\frac{1}{N^{\mathrm{slab}}} \sum_{i \in \Gamma_{\nu}} \sum_{i^{\prime} \in \Gamma_{\nu}^{\prime}} \exp \left(-l \vec{q} \cdot\left(\vec{r}_{i^{\prime}}-\vec{r}_{i}\right)_{x y}\right)\right\rangle$,

involving the projected two-dimensional in-plane vectors $(\vec{r})_{x y}$ and $\vec{q}$. In order to obtain an expression similar to equation (5), we have to contract the coordinates of the static structure factor along the $z$ direction into the wall plane. We start from an in-plane Fourier transform of the total correlation function [45], defined by a Hankel transform via

$$
h_{\nu \nu^{\prime}}^{(2)}\left(z, z^{\prime}, q\right)=\int h_{\nu \nu^{\prime}}^{(2)}\left(z, z^{\prime}, r\right) \mathrm{e}^{-l \vec{q} \cdot(\vec{r})_{x y}} \mathrm{~d}(\vec{r})_{x y} .
$$

Following Tarazona and co-workers [64], we then define partial transverse structure factors $H_{\nu^{\prime} \nu}$ by contracting the $z^{\prime}$ coordinate in the slab of interest via

$$
H_{\nu^{\prime} \nu}^{\mathrm{slab}}(z, q)=\delta_{\nu \nu^{\prime}}+\int_{\text {slab }} \rho_{\nu^{\prime}}\left(z^{\prime}\right) h_{\nu^{\prime} \nu}\left(z^{\prime}, z, q\right) \mathrm{d} z^{\prime},
$$

which, for a one-component bulk fluid, resembles the structure factor $S_{\nu^{\prime} \nu}(k)$. Similar to the static structure factor, the partial transverse structure factors can be used to construct the transverse particle-particle structure $H_{\mathrm{NN}}(z, q)=\sum_{\nu^{\prime}} \sum_{\nu} H_{\nu^{\prime} \nu}(z, q)$, the particle-charge structure $H_{\mathrm{ZN}}(z, q)=\sum_{\nu^{\prime}} \sum_{\nu} Z_{\nu^{\prime}} H_{\nu^{\prime} \nu}(z, q)$, and the charge-charge structure $H_{\mathrm{ZZ}}(z, q)=\sum_{\nu^{\prime}} \sum_{\nu} Z_{\nu^{\prime}} Z_{\nu} H_{\nu^{\prime} \nu}(z, q)$. Finally, a second contraction along the remaining $z$ coordinate defines an in-plane structure factor

$$
\bar{H}_{\nu^{\prime} \nu}^{\mathrm{slab}}(q)=\frac{1}{N^{\text {slab }}} \int_{\text {slab }} \rho_{\nu}(z) H_{\nu^{\prime} \nu}^{\mathrm{slab}}(z, q) \mathrm{d} z,
$$

which resembles the result from equation (7).

\subsection{Density functional theory}

We apply the framework of (classical) DFT [33, 36] to our primitive model. DFT deals with ensemble averaged concentration (density) profiles $\rho_{\nu}$ of species $\nu$, which describe the particle distributions in a grand canonical ensemble. The equilibrium density profiles $\rho_{\nu}^{\mathrm{eq}}$ minimize the grand canonical energy functional $\Omega\left(T, V, \Psi_{\mathrm{p}}, \Psi_{\mathrm{n}},\left\{\mu_{\nu}\right\} ;\left[\sigma_{\mathrm{p}}, \sigma_{\mathrm{n}},\left\{\rho_{\nu}\right\}\right]\right)$, which (when minimized with respect to $\rho_{\nu}(\vec{r}), \sigma_{\mathrm{p}}$, and $\sigma_{\mathrm{n}}$ ) gives the grand canonical potential of the corresponding physical system with temperature $T$, volume $V$, wall potentials $\Psi_{\mathrm{p}}$ and $\Psi_{\mathrm{n}}$, and chemical potentials $\mu_{\nu}$. Thus, the equilibrium density profiles can be determined from

$$
\left.\frac{\delta \Omega\left[\left\{\rho_{\nu}\right\}\right]}{\delta \rho_{\nu}(\vec{r})}\right|_{\left\{\rho_{\nu}=\rho_{\nu}^{(\mathrm{eq})}\right\}}=0 \quad \forall \nu .
$$

The minimization with respect to the wall charge densities is guaranteed by construction on the basis of equations (3) and (4).

The grand canonical potential $\Omega$ can be obtained from the Helmholtz free energy $F$ via a generalized Legendre transform $\Omega=F-\Psi_{\mathrm{p}} Q_{\mathrm{p}}-\Psi_{\mathrm{n}} Q_{\mathrm{n}}-\sum \mu_{\nu} N_{\nu}$, where the extensive unit charges $Q_{\mathrm{p} / \mathrm{n}}$ and particles numbers $N_{\nu}$ are replaced by the intensive electrostatic potentials $\Psi_{\mathrm{p} / \mathrm{n}}$ and chemical potentials $\mu_{\nu}$. It is convenient to split the corresponding free energy functional $\mathcal{F}\left(T, V, \Psi_{\mathrm{p} / \mathrm{n}},\left\{N_{\nu}\right\} ;\left[\sigma_{\mathrm{p} / \mathrm{n}},\left\{\rho_{\nu}\right\}\right]\right)$ into an ideal gas part $\mathcal{F}^{\text {id }}$ and an excess part $\mathcal{F}^{\text {exc }}$, where the latter includes all particle interactions.

Within DFT, direct pair-correlation functions $c_{\nu \nu^{\prime}}^{(2)}$ immediately follow from a second functional derivative of the excess free energy $[33,36]$, such that 


$$
c_{\nu \nu^{\prime}}^{(2)}\left(\vec{r}, \vec{r}^{\prime}\right)=-\beta \frac{\delta^{2} \mathcal{F}^{\mathrm{exc}}}{\delta \rho_{\nu}(\vec{r}) \delta \rho_{\nu^{\prime}}\left(\vec{r}^{\prime}\right)} .
$$

Then, total correlations are obtained via the Ornstein-Zernike relation in equation (6). In principle, the total correlations can also be obtained from determining the pair distribution function $g_{\nu \nu^{\prime}}^{(2)}\left(\vec{r}, \vec{r}^{\prime}\right)$ around a particle fixed at $\vec{r}$, but this socalled test-particle route is problematic in situations where long-ranged particle interactions are present due to the finite boundaries of a numerically treated system (see also the discussion in [45]). We will therefore follow the compressibility route.

As in our previous work [63], and according to the interaction potential of equation (1), we combine the excess free energy functional from a hard-sphere part $\mathcal{F}^{\mathrm{HS}}$ and a Coulombic part $\mathcal{F}^{\mathrm{C}}$. For the hard-sphere contribution, we apply the so-called White Bear mark II functional [62] from fundamental measure theory [40] in its tensor version [65]. This functional has been shown to provide quantitative results for free energies [66], phase coexistence [43, 44], and pair correlations [45]. For the Coulombic part, we add the ionic mean-field contribution

$$
\begin{aligned}
\mathcal{F}^{\mathrm{C}}\left[\left\{\rho_{\nu}\right\}\right] & =k_{\mathrm{B}} T \frac{V}{2 L} \int_{0}^{L} q(z) \phi(z) \mathrm{d} z \\
& =k_{\mathrm{B}} T \frac{\lambda_{\mathrm{B}}}{2} \int_{V} \int_{V} \frac{q(z) q\left(z^{\prime}\right)}{\left|\vec{r}-\vec{r}^{\prime}\right|} \mathrm{d} \vec{r} \mathrm{~d} \vec{r}^{\prime} .
\end{aligned}
$$

Obviously, additional contributions could be added to correct for the correlations between the pure hard-sphere and Coulombic contributions [63], as derived, for example, within the mean spherical approximation in bulk [53-57]. For inhomogeneous systems, such corrections are still missing, and, since we extract pair correlations from DFT including charges for the first time, we neglect any additional and approximate correction in order to clearly separate the contribution of the Coulombic contributions to the excellently performing hardsphere functional.

Similar to the free energy, the direct correlations $c_{\nu \nu^{\prime}}^{(2)}=c_{\nu \nu^{\prime}}^{\mathrm{HS}}+c_{\nu \nu^{\prime}}^{\mathrm{C}}$ can be split into correlations due to the hardsphere and Coulombic part of the excess free energy. The first contribution, arising from the hard-sphere part, was determined in a recent work [45] by one of us, where it was shown to be in excellent agreement with Brownian dynamics simulations. The Coulombic part immediately follows in analytic form from equations (12) and (13) and reads

$$
c_{\nu \nu^{\prime}}^{\mathrm{C}}\left(\vec{r}, \vec{r}^{\prime}\right)=-\beta \frac{\delta^{2} \mathcal{F}_{\mathrm{C}}}{\delta \rho_{\nu}(\vec{r}) \delta \rho_{\nu^{\prime}}\left(\vec{r}^{\prime}\right)}=-\lambda_{\mathrm{B}} \frac{Z_{\nu} Z_{\nu^{\prime}}}{\left|\vec{r}-\vec{r}^{\prime}\right|} .
$$

Following equation (8), $c_{\nu \nu^{\prime}}^{\mathrm{C}}$ can also be Fourier transformed analytically in the $x y$ plane, resulting in

$$
\begin{aligned}
c_{\nu \nu^{\prime}}^{\mathrm{C}}\left(z, z^{\prime}, q\right) & =-\frac{\lambda_{\mathrm{B}}}{2 \pi} \iint \frac{Z_{\nu} Z_{\nu^{\prime}}}{\left|\vec{r}-\vec{r}^{\prime}\right|} \mathrm{e}^{-l \vec{q} \cdot\left(\vec{r}^{\prime}\right)_{x y}} \mathrm{~d}\left(\vec{r}^{\prime}\right)_{x y} \\
& =-\lambda_{\mathrm{B}} Z_{\nu} Z_{\nu^{\prime}} \frac{1}{q} \mathrm{e}^{-q\left|z-z^{\prime}\right|}
\end{aligned}
$$

with $q=|\vec{q}| \neq 0$. In the limit of vanishing $\Delta z=z^{\prime}-z \rightarrow 0$, equation (16) becomes $-\lambda_{\mathrm{B}} Z_{\nu} Z_{\nu^{\prime}} / q$.

Inserting equation (15) and the hard-sphere contributions $c_{\nu \nu^{\prime}}^{\mathrm{HS}}\left(z, z^{\prime}, q\right)$ into equation (6) leads to

$$
\begin{aligned}
h_{\nu \nu^{\prime}}^{(2)}\left(z, z^{\prime}, q\right)= & c_{\nu \nu^{\prime}}^{(2)}\left(z, z^{\prime}, q\right)+2 \pi \sum_{\lambda} \int_{-\infty}^{\infty} \rho_{\lambda}\left(z^{\prime \prime}\right) h_{\nu \lambda}^{(2)}\left(z, z^{\prime \prime}, q\right) \\
& \times c_{\lambda \nu^{\prime}}^{(2)}\left(z^{\prime \prime}, z^{\prime}, q\right) \mathrm{d} z^{\prime \prime}
\end{aligned}
$$

which we then use together with the explicit form equation (16) to calculate the in-plane structure via equation (9).

\section{Choice of model and parameters}

In order to perform quantitative comparisons, we must first choose the model parameters appropriately. Since this work has been inspired by the findings of Merlet and co-workers $[23,67]$, we will use the Lennard-Jones diameters of the spherical particles in their work as the hard-sphere diameter of our particles. Furthermore, we approximated the shape of their elongated $\mathrm{BMI}^{+}$ion by a sphere of the same volume. Consequently, throughout this work we use ionic diameters $2 R_{+}=0.618294 \mathrm{~nm}$ for the $\mathrm{BMI}^{+}$ions and $2 R_{-}=0.506 \mathrm{~nm}$ for the $\mathrm{PF}_{6}^{-}$ions, having a diameter ratio $R_{-} / R_{+} \approx 0.818$ and a asymmetry $\alpha \approx 0.2919$, which is defined for the ionic volumes $V_{+}$and $V_{-}$via $\alpha=\left(1-V_{+} / V_{-}\right) /\left(1+V_{+} / V_{-}\right)$. We also use the same valencies, $Z_{ \pm}= \pm 0.78 \mathrm{e}$, the temperature $T=400 \mathrm{~K}$, and the wall separation $L=12.32 \mathrm{~nm}$ as in [23, 67]. However, we chose a different relative permittivity and mean concentrations different from $\bar{\rho}_{ \pm}=2.345 \mathrm{~nm}^{-3}$ for the following reasons.

In the equal-size limit of $\alpha \rightarrow 0$, the volume-conserving averaged ionic mean radius $\bar{R} \approx 0.5677 \mathrm{~nm}$ allows to draw an approximate comparison between the described binary system of Merlet and co-workers and the well-studied restricted primitive model of equally-sized ions. With a volume fraction $\eta=4 \pi \bar{R}^{3} \bar{\rho} / 3 \approx 0.15$ and an effective dimensionless temperature $T^{*}=2 \bar{R} / \lambda_{\mathrm{B}} \approx 0.01$, the system with $\varepsilon=1$ would be located in the metastable region of the fluid-solid coexistence $[68,69]$, which most probably is avoided for the binary mixture by the asymmetry of the ion radii. Nonetheless, we have chosen to work at a higher effective temperature $T^{*}$ by varying the relative permittivity between $\varepsilon=1$ and 30 , focusing on $\varepsilon=10$ if not mentioned otherwise. By this choice, our work can be related not only to the ionic liquid $\mathrm{BMI}_{-} \mathrm{PF}_{6}$, which tends to demix from water [70], but also to other ionic electrolytes with higher permittivities, such as diluted ionic liquids or tetraethylammonium tetrafluoroborate $\left(\mathrm{TEA}_{-} \mathrm{BF}_{4}\right)$ solvated in acetonitrile (ACN) [8], which is often used in supercapacitors. Such systems have typical concentrations of about $\bar{\rho}_{ \pm}=0.6 \mathrm{~nm}^{-3}$ $(1 \mathrm{M})$, where the electrodes are still screened well and ionic core repulsions are important $\left(\kappa^{-1} \approx 4.6 \mathrm{~nm}\right)$. We will focus in the work at hand on moderate ionic concentrations of around $2 \mathrm{M}$. Note however that we do not aim to give a realistic model for one specific ionic liquid but rather aim to bring forward the knowledge about microscopic effects involved in the composition of the EDL, which all ionic liquids have in common. 
Table 1. Data as obtained from DFT for systems of several relative permittivities $\varepsilon$ at ionic concentrations $\bar{\rho}_{+/-}=2 \mathrm{M}$.

\begin{tabular}{llllrll}
\hline$\varepsilon(1)$ & $\Psi_{\mathrm{p}}(\mathrm{V})$ & $\Psi_{\mathrm{n}}(\mathrm{V})$ & $\begin{array}{l}\sigma_{\mathrm{p}} \\
\left(\mathrm{e} \mathrm{nm}^{-2}\right)\end{array}$ & $\begin{array}{l}\sigma_{\mathrm{n}} \\
\left.(\mathrm{e} \mathrm{nm})^{-2}\right)\end{array}$ & $\begin{array}{l}N_{+} \\
\left(1 / \mathrm{nm}^{2}\right)\end{array}$ & $\begin{array}{l}N_{-} \\
\left(1 / \mathrm{nm}^{2}\right)\end{array}$ \\
\hline 30 & 0.0 & -0.0 & 0.023 & 0.023 & 14.46 & 14.52 \\
30 & 0.437 & -0.563 & 1.775 & -1.775 & 15.09 & 15.11 \\
10 & 0.0 & -0.0 & 0.019 & 0.019 & 14.46 & 14.51 \\
10 & 0.087 & -0.113 & 0.156 & -0.156 & 14.49 & 14.49 \\
10 & 0.1 & -0.1 & 0.177 & -0.136 & 14.47 & 14.52 \\
10 & 0.459 & -0.541 & 0.777 & -0.777 & 14.67 & 14.68 \\
10 & 0.5 & -0.5 & 0.848 & -0.720 & 14.60 & 14.78 \\
2 & 0.0 & -0.0 & 0.011 & 0.011 & 14.46 & 14.49 \\
1 & 0.0 & -0.0 & 0.008 & 0.008 & 14.45 & 14.48 \\
\hline
\end{tabular}

Note: For each system either the electrostatic wall potentials $\Psi_{\mathrm{p} / \mathrm{n}}$ at the positive anode and the negatively charged cathode sum up to zero, or the wall charge densities $\sigma_{\mathrm{p} / \mathrm{n}}$ sum up to zero. In addition, the total numbers $N_{+/}$ of ions of each species + and - in the system are given per unit area of the lateral extension.

In this work, we test our DFT results against MD simulations of charged pseudo hard-sphere particles. Details of the simulation method can be found in previous work [63]. The simulations were performed using the ESPResSo package [71]. There are three notable methodological differences between the simulations and the theory. First, in the simulation we sample the canonical ensemble and not the grandcanonical one, second, we assume transverse symmetry in our theory, and third, we impose constant charge densities and not voltage on the electrodes. To match the theory and simulation setups we use as input for the simulations the particle number densities and surface charges obtained from the DFT calculations (see for example table 1). This is justified by the fact that, first, there is a large bulk-like region in the capacitor, and second, we find that the simulations reproduce accurately the theoretically predicted densities. Furthermore, we performed bulk simulations of the ionic liquid and found excellent match between the simulations and theory. Lastly, we also verified that the correct wall potentials are reproduced in the simulations.

\section{Out-of-plane structure and charging effects}

We first compare the out-of-plane EDL structure resulting from the theory and the MD simulations. In figure 2 we show typical ionic concentration profiles for two systems with a reference bulk concentration of $2 \mathrm{M}$. The electrodes are charged asymmetrically up to potentials (a) $\pm 0.1 \mathrm{~V}$ and (b) $\pm 0.5 \mathrm{~V}$. It is obvious from the profiles that the layering of ions in general is captured in our theory even at high packing and high potentials, but especially the alternating layering of differently charged ions is clearly underestimated. The DFT anion and cation profiles seem to 'stick together' too strongly (see red and green curves in comparison to red and green symbols), while our MD simulations and previous work show alternating layers of both species for high potentials and concentrations $[15,18,19,51,67,72]$. The reason is most probably that the mean-field description of charges from
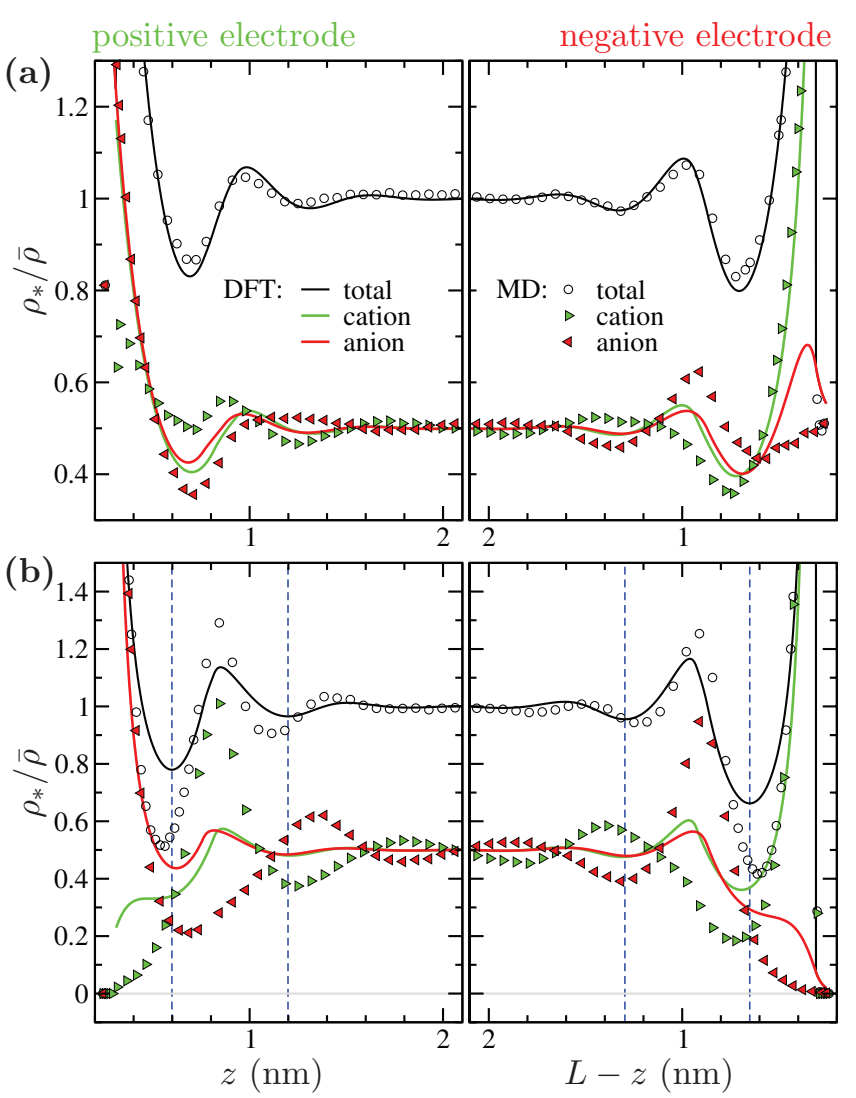

Figure 2. Comparison between DFT results (curves) and MD computer simulations (symbols) for the concentration profiles $\rho_{*}$, where $*$ is a place holder for cations, anions, and the sum of both. The profiles are normalized to the total concentration $\bar{\rho}=\bar{\rho}_{+}+\bar{\rho}$ of ions in bulk. Results are shown for a system with temperature $T=400 \mathrm{~K}$, permittivity $\varepsilon=10$, and bulk concentration $\bar{\rho}_{+/-}=2$ $\mathrm{M}$, at two electrostatic wall potentials (a) $\Psi_{\mathrm{p}}=-\Psi_{\mathrm{m}}=0.1 \mathrm{~V}$ and (b) $0.5 \mathrm{~V}$. The plots show regions in the vicinity of the system walls, which are located at $z=0 \mathrm{~nm}$ and $z=L=12.32 \mathrm{~nm}$. Additionally, panel (b) shows the positions of the first two minima in the total concentration profiles $\bar{\rho}$ next to the wall, which are marked by vertical dashed lines, located at $z=0.598 \mathrm{~nm}, 1.198 \mathrm{~nm}$, $L-1.296 \mathrm{~nm}$, and $L-0.601 \mathrm{~nm}$.

equation (13) is decoupled from the hard-core repulsion [51], which overestimates the Coulombic attraction between ionic cores at small distances, where particles overlap.

The 'sticking effect' of the ionic profiles described above is also visible in figure 3, where we show the profiles for different permittivities between uncharged walls. The DFT results show the typical layering of a binary system, where the small anions reach the walls closer than the larger cations (e.g. $[18,19,51])$. Increasing the dielectric screening by increasing $\varepsilon$ strengthens this layering of anions (arrow up in figure 3), while the layering of the cations is weakened (arrow down in figure 3).

Indeed, the layer of exclusively anions in contact with the wall introduces a local electric field as a counterpart to the mechanical pressure towards the wall. When the potential is forced to vanish at the electrodes, electrical charges are induced on them. Thereby, the charge induced at the wall rises with increasing permittivity, as shown in table 1 . Next to the results, where we initially fixed the electrostatic potentials 


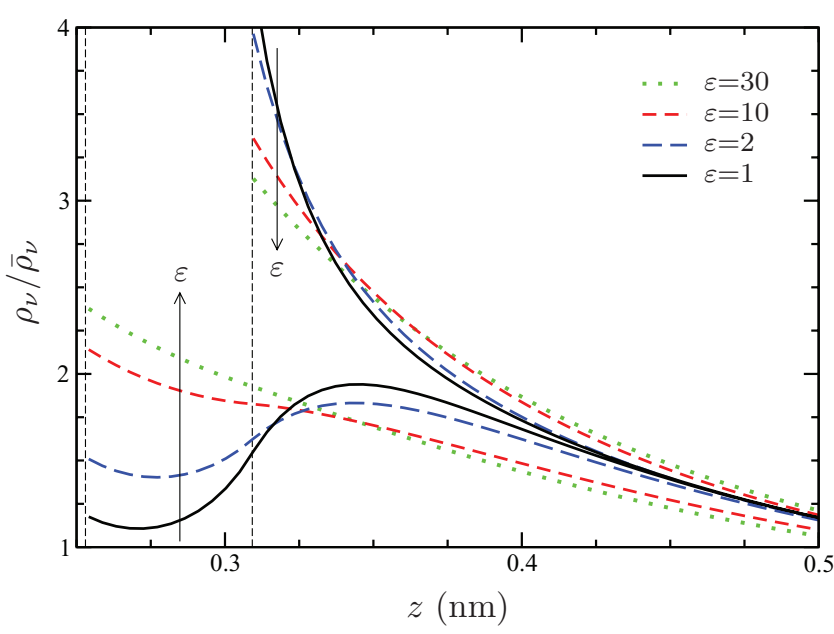

Figure 3. Concentration profiles of the cations and anions next to the left electrode at vanishing potential $\Psi_{\mathrm{p}}$ for four relative permittivities $\varepsilon=1,2,10$, and 30 at an ionic concentration $\bar{\rho}_{+/-}=2 \mathrm{M}$. Due to their sizes, the anions reach the electrode closer than the larger cations, which leads to a separation of the concentration profiles.

$\Psi_{\mathrm{p} / \mathrm{n}}$ at the electrodes, table 1 also shows data for fixed surface charge densities $\sigma_{\mathrm{p} / \mathrm{n}}$ on the electrodes. Since the former is natural for our grand canonic theory, we had to scan over different potentials to force the charges on both plates to add to zero, which models the standard function of a capacitor. Note in this context that we cannot simply shift potentials in our system, because the system is in osmotic contact with a neutral bulk reservoir at zero potential, i.e. the zero-potential point is already chosen in the bulk reservoir.

In figures 4(a) and (b) we show ionic concentration profiles near the positive and negative electrode, respectively, for three applied voltages. When the potential difference $\Psi_{\Delta}=\Psi_{\mathrm{p}}-\Psi_{\mathrm{n}}$ between both electrodes is increased, the ionic charges, which screen the electrodes, reorganize. Recent work indicates that the EDL can be subdivided into layers [19] with ion exchange occurring between them as $\Psi_{\Delta}$ changes. This exchange has also been discussed as the cause of structural in-plane transitions within the EDL when the potential is increased [23]. The local change in the ionic concentrations due to the change in $\Psi_{\Delta}$ is shown in panels (c) and (d). This response function demonstrates how the layers are structured and to which regions ions tend to go to and leave from. For example, the magnification in the inset of figure 4(c) shows that at low potentials the concentration of anions next to the positive electrode increases in all regions when the potential is increased (red solid curve positive for all $z<0.9 \mathrm{~nm}$ ), whereas at higher potentials the change in concentration becomes negative in the region around $z=0.5 \mathrm{~nm}$, corresponding to the minimum around $z=0.6 \mathrm{~nm}$ in the anion concentration profile (red curves) in panel (a). Moreover, figure 4(d) clearly shows how the anions are repelled from the increasingly negative electrode: initially, the rejection is strongest immediately at the wall (red solid curve in panel (d)), but with increasing potential, when the ionic concentration vanishes at the wall (red curves in panel (b)), anions are also rejected from more distant regions where they were attracted to earlier (red dotted curve in panel (d)).
Another salient feature of the EDL that is captured by our DFT is the change in the shape of the concentration peaks when the voltage increases, which indicates a structural transition in the EDL. The cation distributions near the anode in figure 4(a) show that the first peak of the concentration profile decreases with increase of $\Psi_{\Delta}$, vanishing completely for $\Psi_{\Delta}=1.2 \mathrm{~V}$ (black curves). At the same time, the peak shifts to larger $z$ and the (initially) second peak first decreases (dashed curve) and then builds up again (the black solid curve in panel (a) has two peaks, at the wall and at $z \approx 1 \mathrm{~nm}$, the dotted curve has only one peak at $z \approx 0.8 \mathrm{~nm}$ ). The final result is that the first peak (black solid curve at the wall) has been shifted by $0.6 \mathrm{~nm}$ and combined with the second peak (black dotted curve at $z \approx 0.8 \mathrm{~nm}$ ). Furthermore, at large voltages the first layer of cations resides slightly farther from the electrode compared to the second layer of anions (dotted curves in the inset of panel (a)). These structural features of the EDL are very similar to those described by the simulation study of Kirchner et al [24], in which it is argued that these are additional hallmarks of the in-plane ordering of the counter-ion layer adsorbed on the wall. To test this within the framework of DFT we will investigate the in-plane structure factor of the first adsorbed layer in the next section.

The layers of the EDL can be defined in between the minima of the total ionic concentration profile [19], which we show in figure 2(b) by the dashed vertical lines. Here, we ignored the discontinuous minimum which stems from the fact that the smaller anions can approach the wall more closely. The thickness of the layers, which we plot in figure 5(a) for different permittivities, depends on the applied electrostatic wall potentials $\Psi_{\mathrm{p} / \mathrm{n}}$. In figure 5(a), we show the thickness, $L^{*}$, of the first layer, of the second layer, and of the half cell $(L / 2=6.16 \mathrm{~nm})$ at the respective electrode, where $*$ just represents a place holder. Especially at high $\varepsilon$, the thickness of the layers show interesting dependence on the applied potential. In part (b) of figure 5, we plot the number $N_{\nu}^{*}$ of particles relative to bulk in the respective regions. Obviously, ion exchange is most significant in the first layer of ions adjacent to the electrode, in accordance with the spatially resolved results in figures 4(c) and (d). Note, however, that a large response in the ionic concentrations does not correspond to a large change in the total number of particles, but rather to a large change in the local charge density and, hence, in the differential capacitance of the cell (dotted lines remain almost flat in panel (b)).

The differential capacitances per unit area, shown in part (c) of figure 5, are defined as the change of charges per area, $\sigma_{*}$, in a certain subvolume $V_{*}$ of the whole cell due to potentials, hence,

$$
C_{*}^{\mathrm{diff}}=\frac{\partial e \sigma_{*}}{\partial \Psi} .
$$

Applied to the whole cell volume, the voltage dependence of this quantity is of great importance for the properties of charging (super)capacitors, and is therefore often studied in the context of ionic liquids [13, 15, 16, 18-20]. In particular, Kornyshev was the first to discuss the difference between the so-called bell and camel shape of the voltage dependence of the differential capacitance [13], where our results shown in panel (c) are somewhere in between both shapes. For 

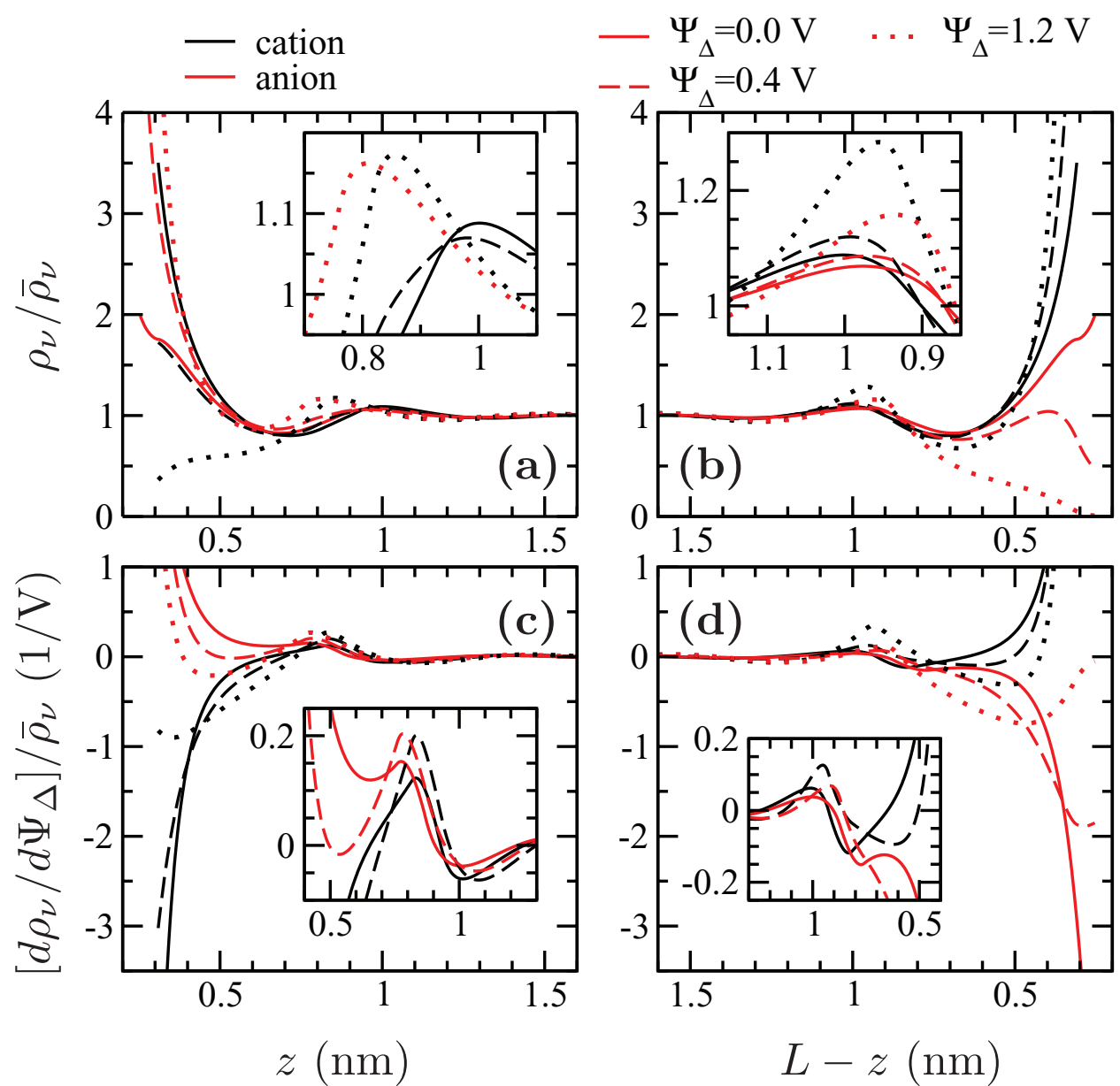

Figure 4. Concentration profiles of cations and anions at (a) the anode and (b) the cathode for different cell potentials $\Psi_{\Delta}=\Psi_{\mathrm{p}}-\Psi_{\mathrm{n}}$ in a system of ionic concentrations $\bar{\rho}_{+/-}=2 \mathrm{M}$ and a relative permittivity $\varepsilon=10$. The insets show magnifications of the second peak in the profiles. The local change in the ionic concentrations with respect to a change in the cell potential is shown in panels (c) and (d), where, again, the insets magnify the regions of the second peaks.

asymmetric particle sizes, these shapes are asymmetrically deformed for positive and negative electrodes [16, 20], as the results in figure 5(c) confirm for the system with $\varepsilon=10$; here, we also provide a comparison between our system's particle asymmetry $\alpha \approx 0.2919$ and two less asymmetric systems, with a smaller and a vanishing $\alpha$, clearly demonstrating the mentioned symmetry for a vanishing $\alpha$ and the asymmetric shift for finite $\alpha$. Interestingly, the differential capacitance of the second layer for $\varepsilon=30$ seems to be almost symmetric, while the first layer shape shows a large asymmetry. Moreover, figure 5(c) demonstrates that the first layer contributes most to the differential capacitance of the respective electrode, especially for small permittivities. Indeed, the contribution of the first layer is almost identical for our system with $\varepsilon=2$ (the lines are on top of each other). Even though the total amount of charge that is stored on the electrodes is higher for higher permittivities (see table 1), the charges are stored in a much smaller volume for low permittivities. For this reason, the permittivity might be decreased in (super)capacitors with very narrow pores in order to maximize the ability to store electrical charges on the electrodes.

In contrast to (super)capacitors, where charge capacities on the electrodes are crucial, molar ion capacities for salt adsorption play an important role in capacitive desalination and capacitive energy extraction $[10,63,73]$. The molar differential capacitance at fixed chemical potential is defined similarly to equation (18) by

$$
C_{\mathrm{mol}, *}^{\mathrm{diff}}=\frac{\partial \mathcal{N}_{*}}{\partial \Psi},
$$

where $\mathcal{N}_{*}$ denotes the total number of particles per electrode area in the volume $V_{*}$ of interest. We plot this measure of particle storage in figure 5(d). Obviously, $C_{\mathrm{mol}, *}^{\mathrm{diff}}$ reaches higher values for higher permittivities, where the repulsion between like-charge ions is better screened. Since the molar differential capacitance is a derivative of the particle number, a vanishing value indicates a minimum in the latter. Interestingly, this minimum is shifted towards negative potentials for increasing asymmetry $\alpha$, such that at the negative electrode the total number of particles is decreased for increasing potentials. This can be understood from figure 4, which shows that the small anions are repelled from the cathode at lower absolute potentials than the cations from the anode. In a simple picture, each cation, adsorbed to the cathode, needs $(1+\alpha) /(1-\alpha)$ anions to leave in order to have enough free volume, as follows 

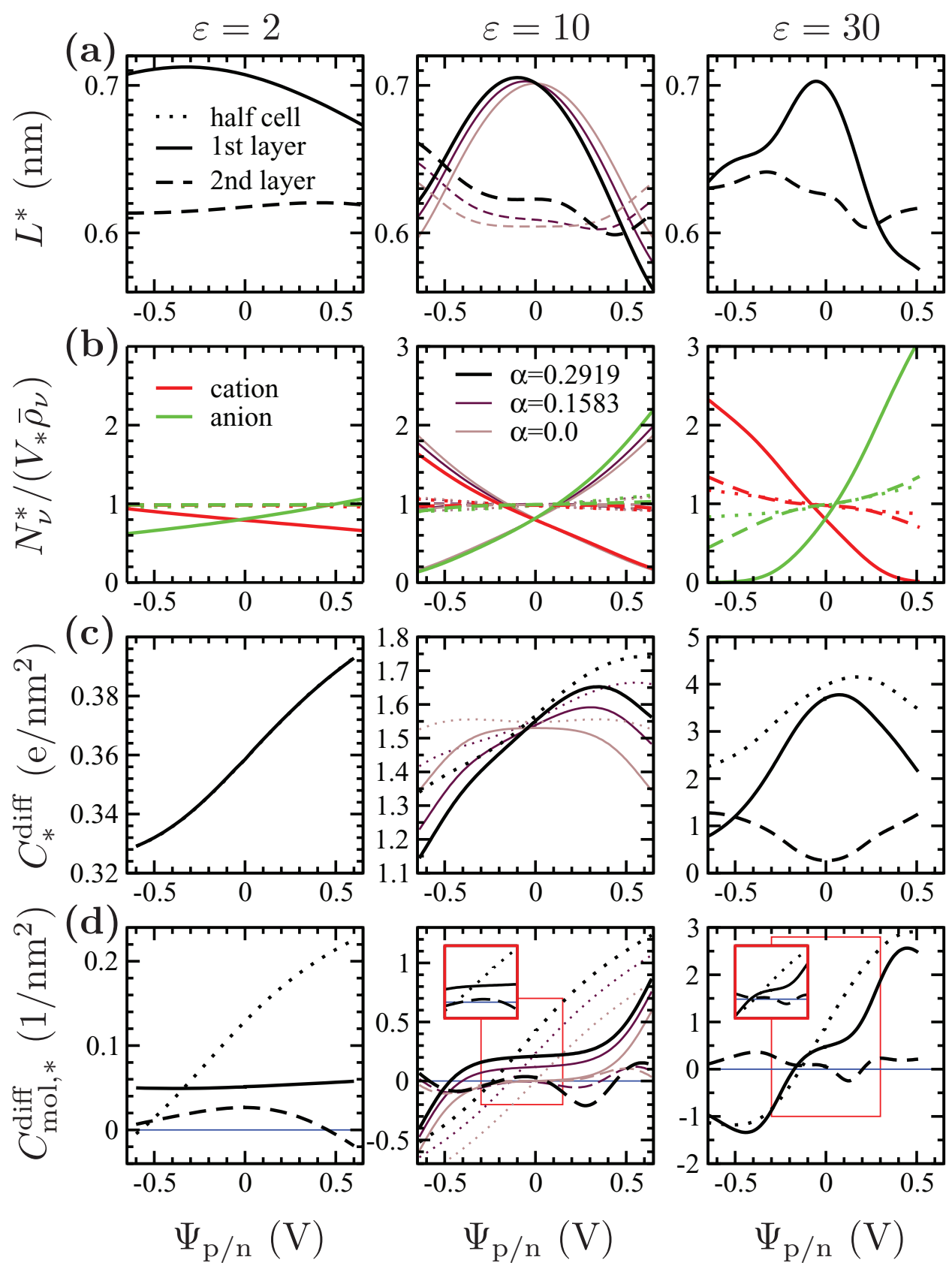

Figure 5. Characteristics of the first two layers at the electrode and of the half cell in terms of (a) their thickness, (b) the amount of particles in relation to bulk, (c) the resulting contribution $C_{*}^{\text {diff }}$ to the differential capacitance, and (d) the contribution $C_{\mathrm{mol}, *}^{\text {diff }}$ to the molar differential capacitance. The results in parts (b), (c), and (d) are separated into cation and anion contributions. The three columns show results for the relative permittivities $\varepsilon=2, \varepsilon=10$, and $\varepsilon=30$, each at ionic concentrations of $\bar{\rho}_{+/-}=2 \mathrm{M}$. In the case of $\varepsilon=10$, data is shown for the additional asymmetries $\alpha=0.1583$ and $\alpha=0.0$ of the ionic species. Furthermore, magnifications in part (d) demonstrate that a decreasing permittivity seems to be similar to a magnification of the graphs at hand, since the magnification at $\varepsilon=30$ is qualitatively similar to the plot at $\varepsilon=10$, where the magnification again is qualitatively similar to the plot at $\varepsilon=2$.

from the definition of $\alpha$ in section 4 . As a consequence, small counterions seem to adsorb better to an electrode than small coions. In conclusion, the effective adsorption of ions requires a detailed study of the adsorption as a function of the ionic sizes and the solvent's permittivity.

\section{In-plane structure of the electric double layer}

In this section, we analyze the partial transverse structure factors $S_{\nu^{\prime} \nu}^{\text {slab }}(q)$ in the first layer next to the anode. Recall that the first layer is defined up to the first minimum of the total concentration profile, $z_{\min }$, ignoring the minimum at $z=R_{+}$due to the small anions reaching closer to the walls. Naturally, $z_{\min }$ depends on the potential $\Psi_{\mathrm{p}}$, as the analysis of the thickness of layers in figure 5(a) shows. Accordingly, we have calculated $H_{\nu^{\prime} \nu}^{\mathrm{slab}}\left(z_{0}, q\right)$ from DFT on the slab interval $\left[R_{-}, z_{\text {min }}\right]$, as explained in equation (9). For technical reasons and in order to compare our DFT-generated in-plane structure to that obtained from our simulations, we have approximated the contraction from equation (10) by $\bar{H}_{\nu^{\prime} \nu}^{\text {slab }}(q)=n_{\nu^{\prime}}^{\text {slab }} H_{\nu^{\prime} \nu}^{\text {slab }}\left(z_{0}, q\right)$ with the mole 

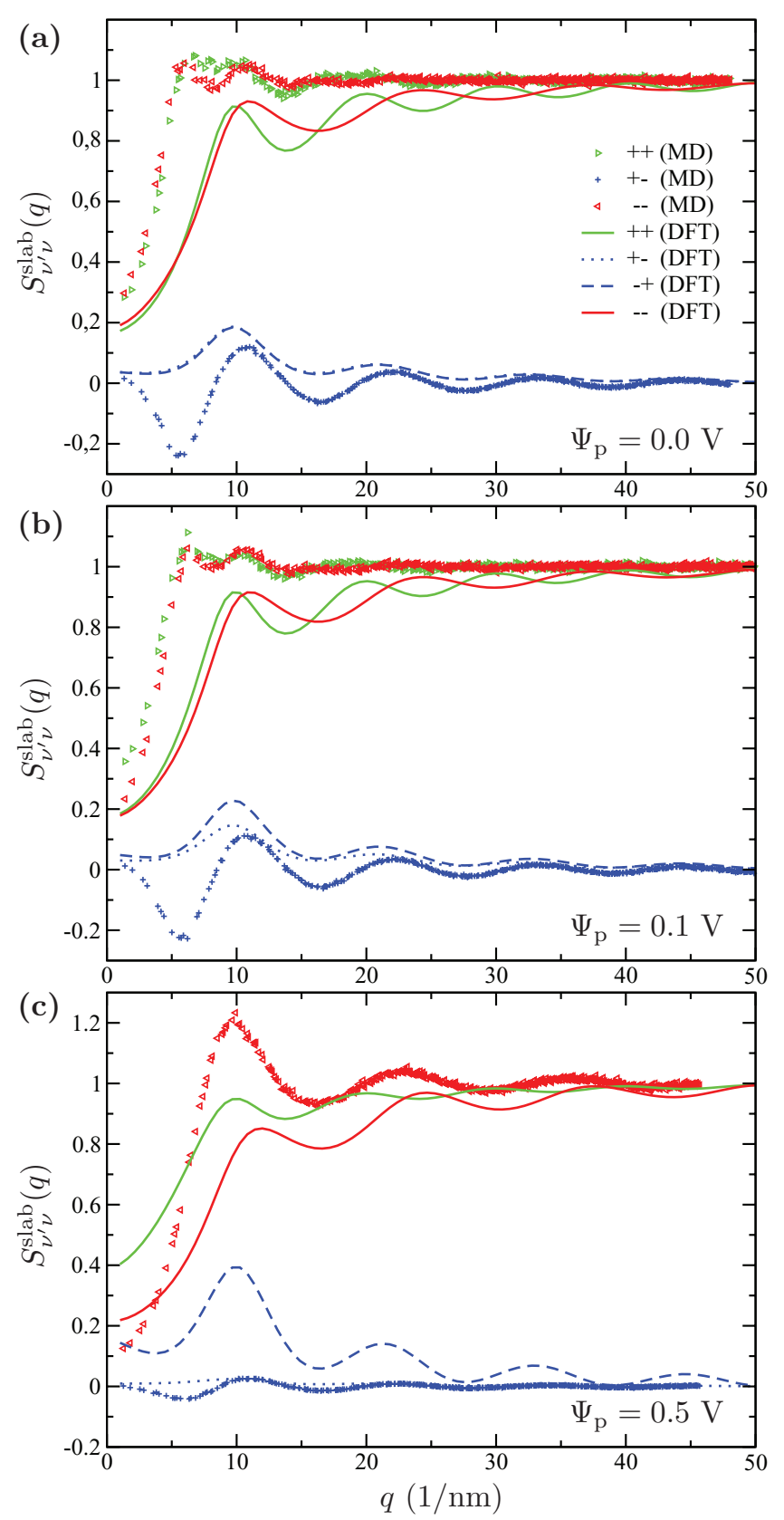

Figure 6. The partial transverse structure factors $S_{\nu^{\prime} \nu}^{\text {slab }}(q)$ in the first layer of ions next to the anode, obtained from both DFT (approximatively) and MD simulations, for electrode potentials (a) $\Psi_{\mathrm{p}}=0.0 \mathrm{~V}$, (b) $\Psi_{\mathrm{p}}=0.1 \mathrm{~V}$, and (c) $\Psi_{\mathrm{p}}=0.5 \mathrm{~V}$. In (c), the ++ contribution from the MD simulations is not shown due to an almost vanishing number of cations, which leads to bad statistics. Note that the diagonal parts $(++$ and --$)$ of the structure factor have been normalized by the mole fraction of the corresponding species to reach comparable large- $q$ limits of 1 .

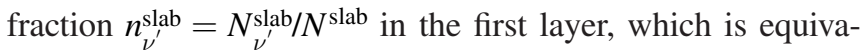
lent to $S_{\nu^{\prime} \nu}^{\text {slab }}(q)$. As a representative position we have chosen $z_{0}=\left(R_{+}+z_{\min }\right) / 2$, such that the slab contains both anions and cations. We did not find any noteworthy differences for other values of $z_{0}$ within the slab. Note that the structure $H_{\nu \nu^{\prime}}^{\text {slab }}\left(z_{0}, q\right)$ still is determined from the complete framework of DFT and the Ornstein-Zernike relation applied to the full inhomogeneous, $z$-dependent density profiles we discussed in this work.
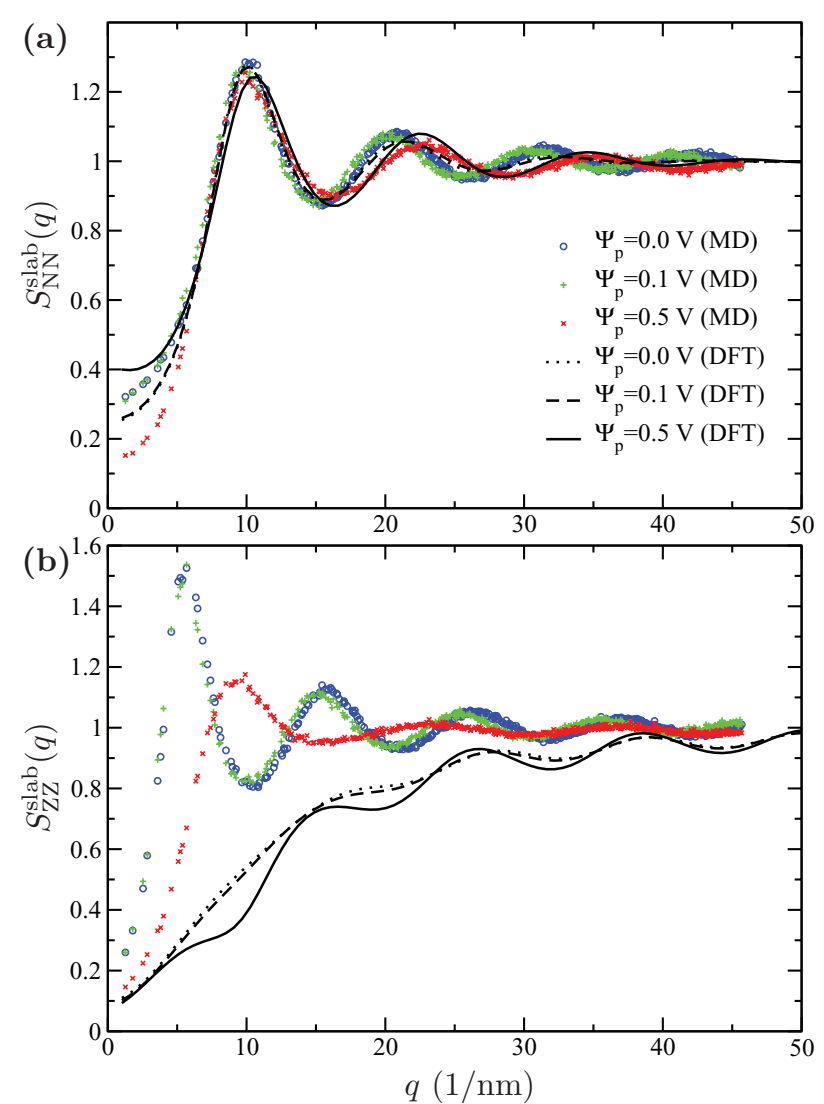

Figure 7. The (a) particle-particle (NN) and (b) charge-charge (ZZ) in-plane structure in the first layer of ions at the positive electrode. The structure is shown for three electrostatic potentials $\Psi_{\mathrm{p}}$ applied to the electrode.

In figure 6, we draw a comparison between both the partial transverse structure factors $S_{\nu^{\prime} \nu}^{\text {slab }}(q)$, approximatively calculated from $n_{\nu^{\prime}}^{\text {slab }} H_{\nu^{\prime} \nu}^{\text {slab }}\left(z_{0}, \circ\right)$ and DFT, and obtained from MD simulations, for three electrode potentials. The first global observation is that there is certainly no quantitative agreement between the DFT results and the simulations. Such a poor agreement should not come as a surprise given (i) the incomplete construction of the free-energy functional by neglecting correlations between the hard-sphere and electrostatic contributions and (ii) the resulting issues in the out-of-plane structure we discussed in the previous section. In particular, we observe in figure 6 that the simulated in-plane structure is more pronounced for the diagonal $(++$ and --$)$ components, reaching the asymptotic large- $q$ limit for smaller values of $q$. This is consistent with the 'sticking' effect of our functional, which gives spurious structure on small length scales and hence on large $q$ 's. The slow large- $q$ decay of the DFT-based structure may well be due to this shortcoming of the DFT. Figure 6 also shows a relatively large difference between panels (a) and (b) compared to panel (c), where the - - correlations obtained by the simulations become very pronounced at $q \sim 10 \mathrm{~nm}^{-1}$, although this can hardly be seen as a sign of a divergence (and hence as a signature of an in-plane phase transition). Our DFT results seem to completely miss this enhanced structural feature in figure 6(c), where simulation results clearly show increased structure at larger $q$ values. This indicates a 
compactification of the first layer of ions in agreement with our previous out-of-plane analysis.

In figures 7(a) and (b) we show the particle-particle (NN) and charge-charge (ZZ) structure, which are linear combinations of the partial in-plane structure factors shown in figure 6 , respectively. Both are presented for the same three potentials as in figure 6 and for both DFT and simulations. The NN structures are all quite similar (except at low $q$ for the high potential simulations) and show a reasonably good agreement between DFT and simulation. This is to be attributed to the high quality of the FMT part of the functional which captures overall packing effects accurately. In contrast, the ZZ structures show a very poor agreement between DFT and simulations, again showing a decay to the large- $q$ limit in DFT that is extremely slow compared to the simulations.

The simulation results in figure 7(b) show a significant shift of the primary peak from $q \sim 6 \mathrm{~nm}^{-1}$ for $\Psi_{\mathrm{p}}=0.1 \mathrm{~V}$ to $q \sim 10$ $\mathrm{nm}^{-1}$ for $\Psi_{\mathrm{p}}=0.5 \mathrm{~V}$, consistent with a very strong adsorption of ionic charge to the electrode surface. As discussed previously, this finding is consistent with the results of Kirchner et al [24] and our findings from section 4. All in all we argue that the present DFT is not sufficient to study the in-plane structure of an ionic liquid in the vicinity of a (highly charged) electrode, and further investigations to correct for the slow large- $q$ decay in the correlations is required.

\section{Concluding discussion}

In this paper we investigated a free-energy functional for a size-asymmetric primitive model of spherical ions in order to describe the in- and out-of-plane structure of an ionic liquid confined in a parallel-plate capacitor by the means of classical DFT. Both the steric repulsions as well as the charges are important in these dense and strongly coupled Coulombic systems. The ionic hard-core repulsions are accurately treated on the basis of a fundamental measure functional whereas the Coulombic interactions are taken into account at a meanfield type Poisson-Boltzmann level. To ensure the isolation of problems we found for the calculation of in-plane structure from DFT, we neglected further (approximate) contributions to the functional, which would correct for correlations between the respected hard-sphere and Coulombic contributions, but which are still unknown for inhomogeneous systems. For several cell voltages we first investigated the ionic density profiles at high concentrations and potentials as a function of the distance from the electrodes, finding reasonable but far-from-perfect agreement with our MD simulations of the same model. The most obvious deviation from our simulation results is a much weaker layering of oppositely charged ions in the EDL, observed from DFT, while general layering and charge exchange in between layers still is captured. Nevertheless, the out-of-plane profiles allow for the calculation of the differential charge and molar capacitances, quantities that are relevant for energy-storage and desalination devices. An interesting issue concerns the possibility of an in-plane structural phase transition associated with anomalies in the differential capacitance as observed recently in simulations [22, 23, 25]. While our out-of-plane results show a hint for this structural transition, the in-plane structure that follows from the direct correlations of our functional gives a very poor account of our simulated in-plane structure. As a main problem, we isolated the slow decay of correlations and connected it with the previously described underestimation of charge layering. However, our work points towards the construction of better functionals, which have to be considered to comprehensively study the observed phenomena in ionic liquids confined in (porous) electrodes. A first candidate is possibly the MSA-corrected functional that was recently used in $[47,63]$. Although we took the asymmetry between cations and anions into account by assigning them different hardsphere diameters, it is also conceivable that the non-spherical shape of some of the ions is a crucial ingredient that needs to be incorporated at the level of the hard-core functional. After all, the rod-like character of some of the ions in real ionic liquids allows for voltage-induced structural changes of the double layer that involve alignment of the ions. Given the practical importance of the systems at hand and the complexity of the required functionals, classical DFT is facing a challenging, interesting, and hopefully bright future to address confined ionic liquids in external potentials.

\section{Acknowledgments}

We thank R Evans for stimulating discussions. This work is part of the D-ITP consortium, a program of the Netherlands Organisation for Scientific Research (NWO) that is funded by the Dutch Ministry of Education, Culture and Science (OCW). We acknowledge financial support by the DFG within priority program SPP 1726 (grant number SP 1382/3-1), from an NWO-VICI grant, and from the European Union under the Marie Skłodowska-Curie grant agreement No. 656327.

\section{References}

[1] Simon P and Gogotsi Y 2008 Nat. Mater. 7845

[2] Vatamanu J and Bedrov D 2015 J. Phys. Chem. Lett. 63594

[3] Zhu Y et al 2011 Science 3321537

[4] Brogioli D 2009 Phys. Rev. Lett. 103058501

[5] Boon N and van Roij R 2011 Mol. Phys. 1091229

[6] Rica R A, Ziano R, Salerno D, Mantegazza F, van Roij R and Brogioli D 2013 Entropy 151388

[7] Hamelers H V M, Schaetzle O, Paz-García J M, Biesheuvel P M and Buisman C J N 2014 Environ. Sci. Technol. Lett. 131

[8] Härtel A, Janssen M, Weingarth D, Presser V and van Roij R 2015 Energy Environ. Sci. 82396

[9] Porada S, Borchardt L, Oschatz M, Bryjak M, Atchison J S, Keesman K J, Kaskel S, Biesheuvel P M and Presser V 2013 Energy Environ. Sci. 63700

[10] Suss M E, Porada S, Sun X, Biesheuvel P M, Yoon J and Presser V 2015 Energy Environ. Sci. 82296

[11] Rogers R D and Seddon K R 2003 Science 302792

[12] Oleksy A and Hansen J-P 2006 Mol. Phys. 1042871

[13] Kornyshev A A 2007 J. Phys. Chem. B 1115545

[14] Fedorov M V and Kornyshev A A 2008 J. Phys. Chem. B 11211868

[15] Georgi N, Kornyshev A and Fedorov M 2010 J. Electroanal. Chem. 649261

[16] Jiang D, Meng D and Wu J 2011 Chem. Phys. Lett. 504153 
[17] Henderson D, Jiang D, Jin Z and Wu J 2012 J. Phys. Chem. B 11611356

[18] Lamperski S, Kaja M, Bhuiyan L B, Wu J and Henderson D 2013 J. Chem. Phys. 139054703

[19] Breitsprecher K, Košovan P and Holm C 2014 J. Phys.: Condens. Matter 26284108

[20] Han Y, Huang S and Yan T 2014 J. Phys.: Condens. Matter 26284103

[21] Kong X, Wu J and Henderson D 2015 J. Colloid Interface Sci. 449130

[22] Merlet C, Salanne M, Rotenberg B and Madden P A 2013 Electrochim. Acta 101262

[23] Merlet C, Limmer D T, Salanne M, van Roij R, Madden P A, Chandler D and Rotenberg B 2014 J. Phys. Chem. C 11818291

[24] Kirchner K, Kirchner T, Ivaništěev V and Fedorov M 2013 Electrochim. Acta 110762

[25] Rotenberg B and Salanne M 2015 J. Phys. Chem. Lett. 64978

[26] Jeon Y, Vaknin D, Bu W, Sung J, Ouchi Y, Sung W and Kim D 2012 Phys. Rev. Lett. 108055502

[27] Griffin J M, Forse A C, Tsai W-Y, Taberna P-L, Simon P and Grey C P 2015 Nat. Mater. 14812

[28] Baus M 1983 Mol. Phys. 48347

[29] Hohenberg P and Kohn W 1964 Phys. Rev. 136 B864

[30] Jones R O 2015 Rev. Mod. Phys. 87897

[31] Mermin N D 1965 Phys. Rev. 137 A1441

[32] Ebner C, Saam W F and Stroud D 1976 Phys. Rev. A 142264

[33] Evans R 1979 Adv. Phys. 28143

[34] Tarazona P, Cuesta J A and Martínez-Ratón Y 2008 Density functional theories of hard particle systems Theory, Simulation of Hard-Sphere Fluids, Related Systems (Lecture Notes in Physics vol 753) ed A Mulero (Berlin: Springer) chapter 7, pp 247-341

[35] Wu J 2006 AIChE J. 521169

[36] Hansen J-P and McDonald I R 2013 Theory of Simple Liquids 4th edn (Amsterdam: Elsevier)

[37] Seaton N A, Walton J P R B and Quirke N 1989 Carbon 27853

[38] Thommes M 2004 Physical adsorption characterization of ordered, amorphous mesoporous materials Nanoporous Materials: Science, Engineering (Series on Chemical Engineering vol 4) ed G Q Lu and X S Zhao (London: Imperial College Press) chapter 11, p 317

[39] Ravikovitch P I and Neimark A V 2006 Langmuir 2211171

[40] Rosenfeld Y 1989 Phys. Rev. Lett. 63980

[41] Roth R 2010 J.Phys.: Condens.Matter 22063102

[42] Marechal M, Korden S and Mecke K 2014 Phys. Rev. E 90042131

[43] Oettel M, Dorosz S, Berghoff M, Nestler B and Schilling T 2012 Phys. Rev. E 86021404

[44] Härtel A, Oettel M, Rozas R E, Egelhaaf S U, Horbach J and Löwen H 2012 Phys. Rev. Lett. 108226101
[45] Härtel A, Kohl M and Schmiedeberg M 2015 Phys. Rev. E 92042310

[46] Alts T, Nielaba P, D'Aguanno B and Forstmann F 1987 Chem. Phys. 111223

[47] MieryTeran L, Suh S H, White H S and Davis H T 1990 J. Chem. Phys. 925087

[48] Patra C N and Ghosh S K 1993 Phys. Rev. E 474088

[49] Biben T, Hansen J P and Rosenfeld Y 1998 Phys. Rev. E 57 R3727

[50] Gillespie D, Nonner W and Eisenberg R S 2003 Phys. Rev. E 68031503

[51] Forsman J, Woodward C E and Trulsson M 2011 J. Phys. Chem. B 1154606

[52] Wang Z, Liu L and Neretnieks I 2011 J. Phys.: Condens. Matter 23175002

[53] Waisman E and Lebowitz J L 1970 J. Chem. Phys. 524307

[54] Høye J S, Lebowitz J L and Stell G 1974 J. Chem. Phys. 613253

[55] Blum L 1975 Mol. Phys. 301529

[56] Stell G and Sun S F 1975 J. Chem. Phys. 635333

[57] Sánchez-Díaz L E, Vizcarra-Rendón A and Medina-Noyola M 2010 J. Chem. Phys. 132234506

[58] Henderson D, Blum L and Lebowitz J L 1979 J. Electroanal. Chem. 102315

[59] Gillespie D 2014 Phys. Rev. E 90052134

[60] Ulander J and Kjellander R 2001 J. Chem. Phys. 1144893

[61] Evans R and Henderson J R 2009 J. Phys.: Condens. Matter 21474220

[62] Hansen-Goos H and Roth R 2006 J. Phys.: Condens.Matter 188413

[63] Härtel A, Janssen M, Samin S and van Roij R 2015 J. Phys.: Condens. Matter 27194129

[64] Tarazona P, Evans R and Marconi U M B 1985 Mol. Phys. 541357

[65] Tarazona P 2000 Phys. Rev. Lett. 84694

[66] Oettel M, Görig S, Härtel A, Löwen H, Radu M and Schilling T 2010 Phys. Rev. E 82051404

[67] Merlet C, Salanne M, Rotenberg B and Madden P A 2011 J. Phys. Chem. C 11516613

[68] Vega C, Abascal J L F and McBride C 2003 J. Chem. Phys. 119964

[69] Hynninen A-P, Leunissen M E, van Blaaderen A and Dijkstra M 2006 Phys. Rev. Lett. 96018303

[70] Chaumont A, Schurhammer R and Wipff G 2005 J. Phys. Chem. B 10918964

[71] Limbach H-J, Arnold A, Mann B A and Holm C 2006 Comput. Phys. Commun. 174704

[72] Mezger M, Roth R, Schrder H, Reichert P, Pontoni D and Reichert H 2015 J. Chem. Phys. 142164707

[73] Rica R A, Ziano R, Salerno D, Mantegazza F and Brogioli D 2012 Phys. Rev. Lett. 109156103 